\title{
MINIMAL LINEAR REPRESENTATIONS OF THE LOW-DIMENSIONAL NILPOTENT LIE ALGEBRAS
}

\author{
J. C. BENJUMEA, J. NÚÑEZ and A. F. TENORIO
}

\begin{abstract}
The main goal of this paper is to compute a minimal matrix representation for each non-isomorphic nilpotent Lie algebra of dimension less than 6. Indeed, for each of these algebras, we search the natural number $n \in \mathbf{N} \backslash\{1\}$ such that the linear algebra $g_{n}$, formed by all the $n \times n$ complex strictly upper-triangular matrices, contains a representation of this algebra. Besides, we show an algorithmic procedure which computes such a minimal representation by using the Lie algebras $\mathfrak{g}_{n}$. In this way, a classification of such algebras according to the dimension of their minimal matrix representations is obtained. In this way, we improve some results by Burde related to the value of the minimal dimension of the matrix representations for nilpotent Lie algebras.
\end{abstract}

\section{Introduction}

Given a nilpotent Lie algebra, it is well-known that there exists $n \in \mathbf{N} \backslash\{1\}$ such that this algebra is isomorphic to a subalgebra of the nilpotent Lie algebra $\mathrm{g}_{n}$ formed by $n \times n$ complex upper-triangular matrices with zeros in the main diagonal (see [8, Theorem 3.6.6]). So, it is interesting to know what is the minimal $n \in \mathbf{N} \backslash\{1\}$ such that a given nilpotent complex Lie algebra can be contained as a subalgebra in $\mathfrak{g}_{n}$ but not in $\mathfrak{g}_{n-1}$.

In this way, the main aim of this paper is not only to get a representation of each low dimensional nilpotent Lie algebra by using Lie algebras $\mathfrak{g}_{n}$, but to compute a representation of these algebras being minimal in the following sense: the given nilpotent Lie algebra is represented by a subalgebra of $g_{n}$ but not by a subalgebra of $g_{n-1}$.

At this respect, Burde [3] studied the minimal dimension $\mu(\mathfrak{g})$ for the representations of a given Lie algebra $g$, although he made it by considering any faithful $\mathfrak{g}$-module and not only the Lie algebras $\mathfrak{g}_{n}$. Consequently, $\mu(\mathfrak{g})$ is less than or equal to the dimension which we are trying to look for.

In other recent papers (for instance, Ghanam et al. [5]), matrix representations were computed for low dimensional nilpotent Lie algebras. However, the representations given in such papers are not usually minimal; indeed, the

Received September 11, 2006. 
minimality of such representations is not studied often. Moreover, although minimal representations have been also previously studied in the literature, we are constraining this study to representations by means of Lie algebras $\mathfrak{g}_{n}$. This approach could be a very natural and novel topic, which runs besides in the opposite direction to the literature in this area.

Benjumea [1] already obtained implicitly representations of the filiform Lie algebras (a subset of nilpotent Lie algebras) of dimension less than six, although the minimality of these representations was not studied. Now, we prove that these representations are minimal and, then, we compare our results with Burde's ones. Besides, the minimal dimension of the representations by using Lie algebras $\mathfrak{g}_{n}$ is obtained for each nilpotent Lie algebras of dimension less than six. Note that the bounds given by Burde [3] do not sometimes supply sufficient information and then we need to prove directly the minimal dimension of the representation.

Another question is to know what Lie algebras have an $n$-dimensional representation. At present, it is unsolved, although Burde obtained some families of Lie algebras satisfying such a property. In this paper we answer this question for low-dimensional nilpotent Lie algebras.

\section{Preliminaries}

In this paper several different types of Lie algebras are dealt: nilpotent, filiform and abelian. Their definitions and main properties can be checked in [6], [8], for instance. We only recall here the following definition: The lower central series (L.C.S.) of a given Lie algebra $g$ is the sequence:

$$
\begin{aligned}
\mathscr{C}^{1}(\mathfrak{g})=\mathfrak{g}, \mathscr{C}^{2}(\mathfrak{g})=[\mathfrak{g}, \mathfrak{g}], \mathscr{C}^{3}(\mathfrak{g})=\left[\mathscr{C}^{2}(\mathfrak{g}), \mathfrak{g}\right], \\
\ldots, \mathscr{C}^{k}(\mathfrak{g})=\left[\mathscr{C}^{k-1}(\mathfrak{g}), \mathfrak{g}\right], \ldots
\end{aligned}
$$

A type of nilpotent Lie algebras is constituted by the $k$-step nilpotent ones, with $k \in \mathbf{N}$. A nilpotent Lie algebra is said $k$-step when $\mathscr{C}^{k+1} \equiv\{0\}$.

Proposition 2.1. Let $\mathrm{g}$ be a Lie algebra and let $\mathrm{g}^{\prime}$ be a Lie subalgebra of g. Then, $\mathscr{C}^{k}\left(\mathfrak{g}^{\prime}\right) \subseteq \mathscr{C}^{k}(\mathfrak{g})$, for all $k \in \mathrm{N}$.

Throughout the paper, we use the following classification of nilpotent Lie algebras of dimension less than 6, which is shown in [6], although the decomposable nilpotent Lie algebras were not included in it: 


\begin{tabular}{|c|c|c|c|}
\hline Dim. & Lie algebra & Law of the algebra & L.C.S. \\
\hline 1 & $n_{1}^{1}$ & abelian & (0) \\
\hline 2 & $\mathfrak{n}_{2}^{1}$ & abelian & (0) \\
\hline \multirow[t]{2}{*}{3} & $\mathfrak{n}_{3}^{1}$ & {$\left[e_{1}, e_{3}\right]=e_{2}$} & $(1,0)$ \\
\hline & $\mathfrak{n}_{3}^{2}$ & abelian & (0) \\
\hline \multirow[t]{3}{*}{4} & $\mathfrak{n}_{4}^{1}$ & {$\left[e_{1}, e_{4}\right]=e_{3},\left[e_{1}, e_{3}\right]=e_{2}$} & $(2,1,0)$ \\
\hline & $\mathfrak{n}_{4}^{2}$ & abelian & (0) \\
\hline & $\mathfrak{n}_{4}^{3}=\mathfrak{n}_{3}^{1} \oplus \mathfrak{n}_{1}^{1}$ & {$\left[e_{1}, e_{3}\right]=e_{2}$} & $(1,0)$ \\
\hline \multirow[t]{9}{*}{5} & $\mathfrak{n}_{5}^{1}$ & {$\left[e_{1}, e_{5}\right]=e_{4},\left[e_{1}, e_{4}\right]=e_{3},\left[e_{1}, e_{3}\right]=e_{2}$} & $(3,2,1,0)$ \\
\hline & $\mathfrak{n}_{5}^{2}$ & {$\left[e_{1}, e_{5}\right]=e_{4},\left[e_{1}, e_{4}\right]=e_{3},\left[e_{1}, e_{3}\right]=\left[e_{4}, e_{5}\right]=e_{2}$} & $(3,2,1,0)$ \\
\hline & $\mathfrak{n}_{5}^{3}$ & {$\left[e_{1}, e_{5}\right]=e_{4},\left[e_{1}, e_{4}\right]=e_{3},\left[e_{4}, e_{5}\right]=e_{2}$} & $(3,2,0)$ \\
\hline & $\mathfrak{n}_{5}^{4}$ & {$\left[e_{1}, e_{5}\right]=e_{4},\left[e_{1}, e_{3}\right]=e_{2},\left[e_{2}, e_{3}\right]=e_{4}$} & $(2,1,0)$ \\
\hline & $\mathfrak{n}_{5}^{5}$ & {$\left[e_{1}, e_{5}\right]=e_{4},\left[e_{1}, e_{3}\right]=e_{2}$} & $(2,0)$ \\
\hline & $\mathfrak{n}_{5}^{6}$ & {$\left[e_{1}, e_{5}\right]=e_{4},\left[e_{2}, e_{3}\right]=e_{4}$} & $(1,0)$ \\
\hline & $n_{5}^{7}$ & abelian & (0) \\
\hline & $\mathfrak{n}_{5}^{8}=\mathfrak{n}_{4}^{1} \oplus \mathfrak{n}_{1}^{1}$ & {$\left[e_{1}, e_{4}\right]=e_{3},\left[e_{1}, e_{3}\right]=e_{2}$} & $(2,1,0)$ \\
\hline & $\mathfrak{n}_{5}^{9}=\mathfrak{n}_{3}^{1} \oplus \mathfrak{n}_{2}^{1}$ & {$\left[e_{1}, e_{3}\right]=e_{2}$} & $(1,0)$ \\
\hline
\end{tabular}

A linear or matrix algebra is that whose vectors are matrices and the Lie bracket is defined by the commutator $[a, b]=a \cdot b-b \cdot a$. So, they are subalgebras of some general linear algebra $\mathfrak{g l}(\mathrm{C}, n)$, formed by $n \times n$ complex regular matrices. When dealing with Lie algebras, linear algebras are used since they are the simplest and that every Lie algebra is isomorphic to some linear algebra according to Ado's Theorem.

Given a Lie algebra $\mathfrak{g}$, a representation of $\mathfrak{g}$ in $\mathrm{C}^{n}$ is a Lie algebra homomorphism $\phi: \mathfrak{g} \rightarrow \mathfrak{g l}\left(\mathrm{C}^{n}\right)=\mathfrak{g l}(\mathrm{C}, n)$. The natural integer $n$ is called the dimension of this representation.

Representations can be also defined by using arbitrary $n$-dimensional vector spaces $V$ (see [4], [6]). In such a case, a representation would be a Lie algebra homomorphism from $\mathfrak{g}$ to the Lie algebra $\mathfrak{g} l(V)$ of endomorphisms of the vector space $V$, which is called g-module. Obviously, there exists a unique $n \in \mathrm{N}$ such that $V$ is isomorphic to $\mathrm{C}^{n}$ and, hence, such that $\mathfrak{g l}(V)$ is isomorphic to $\mathfrak{g l}(\mathrm{C}, n)$. So, it is sufficient to consider representations in $\mathrm{C}^{n}$. Besides, we only use faithful representations, due that they allow us to consider the studied Lie algebra as a Lie subalgebra of $\mathfrak{g l}(\mathrm{C}, n)$. In this sense, every Lie algebra can be represented in some Lie algebra $\mathfrak{g l}(\mathrm{C}, n)$. 
For nilpotent Lie algebras, their representations can be obtained by using the Lie algebras $\mathfrak{g}_{\mathfrak{n}}$ (see [8]). For $n \in \mathbf{N} \backslash\{1\}$, the Lie algebra $\mathfrak{g}_{n}$ is the subalgebra of $g \mathfrak{l}(\mathrm{C}, n)$ formed by the following $n \times n$ upper-triangular matrices:

$$
\left(\begin{array}{cccccc}
0 & x_{1,2} & x_{1,3} & \cdots & x_{1, n-1} & x_{1, n} \\
0 & 0 & x_{2,3} & \cdots & x_{2, n-1} & x_{2, n} \\
0 & 0 & 0 & \cdots & x_{3, n-1} & x_{3, n} \\
\vdots & \vdots & \vdots & \ddots & \vdots & \vdots \\
0 & 0 & 0 & \cdots & 0 & x_{n-1, n} \\
0 & 0 & 0 & \cdots & 0 & 0
\end{array}\right) .
$$

A basis $\left\{e_{i, j} \mid 1 \leq i \leq n-1 \wedge i+1 \leq j \leq n\right\}$ of $g_{n}$ can be computed in such a way that its nonzero brackets are (see [7]):

$$
\left[e_{i, h}, e_{h, k}\right]=e_{i, k}, \quad h=1, \ldots, k-1 ; \quad i=1, \ldots, h-1,
$$

and, hence, the lower central series of $\mathfrak{g}_{n}$ is:

$$
\left(\operatorname{dim}\left(\mathfrak{g}_{n-1}\right), \operatorname{dim}\left(\mathfrak{g}_{n-2}\right), \ldots, \operatorname{dim}\left(\mathfrak{g}_{2}\right), 0\right)
$$

Given a nilpotent Lie algebra $g$, we are interested in computing the minimal value $n$ (which will be denoted by $\widehat{\mu}(\mathfrak{g})$ ) such that $g_{n}$ contains $\mathfrak{g}$. It is easy to prove that this value is an invariant of $g$ whose expression is:

$$
\widehat{\mu}(\mathfrak{g})=\min \left\{n \in \mathrm{N} \mid \exists \text { subalgebra of } \mathfrak{g}_{n} \text { isomorphic to } \mathfrak{g}\right\} \text {. }
$$

Observe that this concept is related with another introduced by Burde [3] for an arbitrary Lie algebra $g$, not necessarily nilpotent. We are referring to the invariant $\mu(\mathfrak{g})$, defined as:

$$
\mu(\mathfrak{g})=\min \{\operatorname{dim}(M) \mid M \text { is a faithful } \mathfrak{g} \text {-module }\}
$$

This value is also equal to the minimal value $n$ such that $\mathfrak{g l}\left(\mathrm{C}^{n}\right)$ contains a subalgebra isomorphic to $\mathfrak{g}$.

Obviously, the inequality $\mu(\mathfrak{g}) \leq \widehat{\mu}(\mathrm{g})$ is satisfied. So, an upper bound for $\widehat{\mu}(\mathfrak{g})$ is also one for $\mu(\mathfrak{g})$. Analogously, if a lower bound is got for $\mu(\mathfrak{g})$, the same value is a lower bound for $\widehat{\mu}(\mathfrak{g})$.

There exist several well-known results about the invariant $\mu(\mathfrak{g})$. Some of them supply the exact value of $\mu(\mathfrak{g})$ and, therefore, imply a lower bound for $\widehat{\mu}(\mathfrak{g})$. The exact value of $\mu(\mathfrak{g})$ is already computed for abelian algebras and filiform Lie algebras (under certain conditions). These computations of $\mu(\mathfrak{g})$ were obtained in [3] and we reproduce some of them: 
Proposition 2.2. Let $\mathrm{g}$ be a Lie algebra of dimension $n$.

(1) If $g$ is abelian, then $\mu(\mathfrak{g})=\lceil 2 \sqrt{n-1}\rceil$.

(2) If $\mathfrak{g}$ is 2-step nilpotent with 1-dimensional center, then $\mu(\mathfrak{g})=\frac{n+3}{2}$.

(3) If $g$ is complex filiform such that $n<10$, then $\mu(\mathfrak{g})=n$.

(4) If $\mathfrak{g}=\mathfrak{S}_{k}$ (Heisenberg algebra of dimension $\left.2 k+1\right)$, then $\mu(\mathfrak{g})=k+2$.

Trivially, the values of $\mu(g)$ given in Proposition 2.2 are lower bounds for $\widehat{\mu}(g)$ in their respective cases. However, fixed an arbitrary nilpotent Lie algebra $\mathfrak{g}$, upper bounds of $\mu(\mathfrak{g})$ have been only obtained, although these are not necessarily upper bounds of $\widehat{\mu}(\mathrm{g})$. Next, we indicate one of these bounds (see [2]) corresponding to the nilpotent Lie algebras considered in this paper.

Proposition 2.3. Let $\mathrm{g}$ be a nilpotent Lie algebra of dimension $n$ less than 8. Then $\mu(\mathfrak{g}) \leq n+1$.

\section{Computing Minimal Matrix Representations of Nilpotent Lie Algebras}

In this section we show how to obtain minimal matrix representations of nilpotent Lie algebras by using the Lie algebras $\mathfrak{g}_{n}$. These representations will be obtained for each nilpotent Lie algebra of dimension less than 6 in $C^{n}$. As we have already mentioned, these representations are interesting because they are minimal in the following sense: finding a matrix representation of a Lie algebra $g$ in $g_{n}$ means that it will not be possible to find another representation of $\mathfrak{g}$ in $\mathfrak{g}_{n-1}$. So, we will say that $n$ will be the minimal dimension for the matrix representations of $\mathfrak{g}$.

Proposition 2.2 gives the exact value of the minimal dimension for representations of some types of Lie algebras. However, such a minimal representation does not have to be obtained by matrices in $\mathfrak{g}_{n}$. In consequence, Proposition 2.2 only gives a lower bound of the minimal dimension for representations of a nilpotent Lie algebra by using matrix algebras $\mathfrak{g}_{n}$. In this way, we have to determine if the lower bound given in such a proposition is reached or if the minimal dimension is greater than the lower bound.

To do it, we are going to give a step by step explanation of the method used to determine a minimal representation (by using matrix algebras $\mathfrak{g}_{n}$ ) of a given nilpotent Lie algebra $\mathfrak{m}$ of dimension $r$ :

(1) Firstly, we use the lower bound obtained starting from Proposition 2.2 and we rule out the Lie algebras $\mathfrak{g}_{k}$ such that $k$ is less than such a lower bound. Analogously, we also rule out those Lie algebras $\mathfrak{g}_{k}$ whose lower central series is not compatible with the corresponding one of $\mathfrak{m}$, in virtue of Proposition 2.1. 
(2) Once we have ruled out the Lie algebras $g_{n}$ indicated in the previous step, we compute the first Lie algebra $\mathfrak{g}_{n}$ containing a subalgebra isomorphic to the nilpotent Lie algebra considered. So, let us suppose that the Lie algebras $\mathfrak{g}_{k}$, for $k=2, \ldots, n$, are not suitable to represent $\mathfrak{m}$. Then, we study if the Lie algebra $g_{n+1}$ is. To do it, we consider an arbitrary basis of a $r$-dimensional Lie subalgebra in $\mathfrak{g}_{n+1}$ and we impose the law of $\mathfrak{m}$ in that subalgebra. By equalling component by component in the obtained conditions, an equation system is got. Every solution of this system can be interpreted as the coefficients of an $r$-dimensional Lie subalgebra of $\mathfrak{g}_{n+1}$ isomorphic to $\mathfrak{m}$.

If such a system is not compatible, we can assert that it is not possible to find in $\mathfrak{g}_{n+1}$ a Lie subalgebra isomorphic to $\mathfrak{m}$. Therefore, $\mathfrak{m}$ cannot be represented in $\mathfrak{g}_{n+1}$ and we would repeat the described procedure with the Lie algebra $\mathfrak{g}_{n+2}$. When we obtain a compatible equation system, we obtain a minimal representation of $\mathfrak{m}$.

The number of solutions in the system depends on the particular Lie algebra studied in each case. This is because such solutions have to verify other additional restrictions to the ones given by the system, namely, the vectors obtained with the solution have to form a basis of the subalgebra. In any case, the solutions of the system can be categorized into a finite number of cases (each of them with a infinite number of solutions). Some of these cases do not lead to a basis of a subalgebra in the studied Lie algebra.

In order to choose a particular solution of the previous system, we search one whose number of unknowns equal to zero is the greatest. Besides, we also try to give a representative of each maximal abelian subalgebra having the minimal number of summand in the basic vectors. Such a representative is, in our opinion, the natural choice to represent the abelian subalgebra.

In this way, we will be able to find minimal representations of all the nilpotent Lie algebras of a fixed dimension provided a classification of these algebras is available, although such classifications are not known in the case of dimensions greater than seven. So, this method can be reasonably applied to get results for any higher dimensional nilpotent Lie algebra because new computational problems would not appear, except those related to the increasing of the number of unknowns and equations. In fact, let us note that the degree of polynomials involved is the same for all cases.

Next, as an example, we apply the method to a particular nilpotent Lie algebra, namely, the Lie algebra $\mathfrak{n}_{5}^{4}$ and, in the following two subsections, we show the results obtained by the application of this method to nilpotent Lie algebras of dimension less than 5 and equal to 5 , respectively, whose classifications are indeed known. 
EXAMPLE 3.1. In this example, we compute the minimal representation of the Lie algebra $\mathfrak{n}_{5}^{4}$ by applying the method explained before. We have chosen this algebra because the representation of it given in [5] was not minimal. Indeed, the dimension of its matrix representation is reduced one unit, since our representation has dimension 4 and the given in [5] has dimension 5.

(1) Inclusions in lower central series according to Proposition 2.1:

$$
\begin{aligned}
\left\langle e_{2}, e_{4}\right\rangle & =\mathscr{C}^{2}\left(\mathfrak{n}_{5}^{4}\right) \subseteq \mathscr{C}^{2}\left(\mathfrak{g}_{4}\right)=\left\langle e_{1,3}, e_{1,4}, e_{2,4}\right\rangle ; \\
\left\langle e_{4}\right\rangle & =\mathscr{C}^{3}\left(\mathfrak{n}_{5}^{4}\right) \subseteq \mathscr{C}^{3}\left(\mathfrak{g}_{4}\right)=\left\langle e_{1,4}\right\rangle .
\end{aligned}
$$

(2) We consider an arbitrary basis $\left\{e_{i}\right\}_{i=1}^{5}$ of a 5-dimensional Lie subalgebra of $g_{4}$. The expression of these basis vectors is:

$e_{i}=\lambda_{1,2}^{i} e_{1,2}+\lambda_{1,3}^{i} e_{1,3}+\lambda_{1,4}^{i} e_{1,4}+\lambda_{2,3}^{i} e_{2,3}+\lambda_{2,4}^{i} e_{2,4}+\lambda_{3,4}^{i} e_{3,4}, \quad \forall i=1, \ldots, 5$.

According to the lower central series of $\mathfrak{n}_{5}^{4}$, we can simplify the expressions of $e_{2}$ and $e_{4}$ :

$$
e_{2}=\lambda_{1,3}^{2} e_{1,3}+\lambda_{1,4}^{2} e_{1,4}+\lambda_{2,4}^{2} e_{2,4} ; \quad e_{4}=\lambda_{1,4}^{4} e_{1,4}
$$

(3) Now, all the brackets $\left[e_{i}, e_{j}\right]$ are computed:

$$
\begin{aligned}
& {\left[e_{1}, e_{2}\right]=\left(\lambda_{1,2}^{1} \lambda_{2,4}^{2}-\lambda_{3,4}^{1} \lambda_{1,3}^{2}\right) e_{1,4} \text {; }} \\
& {\left[e_{1}, e_{3}\right]=\left(\lambda_{1,2}^{1} \lambda_{2,3}^{3}-\lambda_{2,3}^{1} \lambda_{1,2}^{3}\right) e_{1,3}+\left(\lambda_{2,3}^{1} \lambda_{3,4}^{3}-\lambda_{3,4}^{1} \lambda_{2,3}^{3}\right) e_{2,4}} \\
& +\left(\lambda_{1,2}^{1} \lambda_{2,4}^{3}+\lambda_{1,3}^{1} \lambda_{3,4}^{3}-\lambda_{2,4}^{1} \lambda_{1,2}^{3}-\lambda_{3,4}^{1} \lambda_{1,3}^{3}\right) e_{1,4} ; \\
& {\left[e_{1}, e_{4}\right]=0} \\
& {\left[e_{1}, e_{5}\right]=\left(\lambda_{1,2}^{1} \lambda_{2,3}^{5}-\lambda_{2,3}^{1} \lambda_{1,2}^{5}\right) e_{1,3}+\left(\lambda_{2,3}^{1} \lambda_{3,4}^{5}-\lambda_{3,4}^{1} \lambda_{2,3}^{5}\right) e_{2,4}} \\
& +\left(\lambda_{1,2}^{1} \lambda_{2,4}^{5}+\lambda_{1,3}^{1} \lambda_{3,4}^{5}-\lambda_{2,4}^{1} \lambda_{1,2}^{5}-\lambda_{3,4}^{1} \lambda_{1,3}^{5}\right) e_{1,4} ; \\
& {\left[e_{2}, e_{3}\right]=\left(\lambda_{1,3}^{2} \lambda_{3,4}^{3}-\lambda_{2,4}^{2} \lambda_{1,2}^{3}\right) e_{1,4} \text {; }} \\
& {\left[e_{2}, e_{4}\right]=0} \\
& {\left[e_{2}, e_{5}\right]=\left(\lambda_{1,3}^{2} \lambda_{3,4}^{5}-\lambda_{2,4}^{2} \lambda_{1,2}^{5}\right) e_{1,4} ;} \\
& {\left[e_{3}, e_{4}\right]=0} \\
& {\left[e_{3}, e_{5}\right]=\left(\lambda_{1,2}^{3} \lambda_{2,3}^{5}-\lambda_{2,3}^{3} \lambda_{1,2}^{5}\right) e_{1,3}+\left(\lambda_{2,3}^{3} \lambda_{3,4}^{5}-\lambda_{3,4}^{3} \lambda_{2,3}^{5}\right) e_{2,4}} \\
& +\left(\lambda_{1,2}^{3} \lambda_{2,4}^{5}+\lambda_{1,3}^{3} \lambda_{3,4}^{5}-\lambda_{2,4}^{3} \lambda_{1,2}^{5}-\lambda_{3,4}^{3} \lambda_{1,3}^{5}\right) e_{1,4} \text {; } \\
& {\left[e_{4}, e_{5}\right]=0 \text {. }}
\end{aligned}
$$


(4) By imposing the law of $\mathfrak{n}_{5}^{4}$ to these brackets, the following equation system is got:

$$
\begin{aligned}
0 & =\lambda_{1,2}^{1} \lambda_{2,4}^{2}-\lambda_{3,4}^{1} \lambda_{1,3}^{2} ; & \lambda_{1,3}^{2} & =\lambda_{1,2}^{1} \lambda_{2,3}^{3}-\lambda_{2,3}^{1} \lambda_{1,2}^{3} ; \\
\lambda_{1,4}^{2} & =\lambda_{1,2}^{1} \lambda_{2,4}^{3}+\lambda_{1,3}^{1} \lambda_{3,4}^{3}-\lambda_{2,4}^{1} \lambda_{1,2}^{3}-\lambda_{3,4}^{1} \lambda_{1,3}^{3} ; & \lambda_{2,4}^{2} & =\lambda_{2,3}^{1} \lambda_{3,4}^{3}-\lambda_{3,4}^{1} \lambda_{2,3}^{3} ; \\
\lambda_{1,4}^{4} & =\lambda_{1,2}^{1} \lambda_{2,4}^{5}+\lambda_{1,3}^{1} \lambda_{3,4}^{5}-\lambda_{2,4}^{1} \lambda_{1,2}^{5}-\lambda_{3,4}^{1} \lambda_{1,3}^{5} ; & 0 & =\lambda_{1,2}^{1} \lambda_{2,3}^{5}-\lambda_{2,3}^{1} \lambda_{1,2}^{5} ; \\
0 & =\lambda_{2,3}^{1} \lambda_{3,4}^{5}-\lambda_{3,4}^{1} \lambda_{2,3}^{5} ; & \lambda_{1,4}^{4} & =\lambda_{1,3}^{2} \lambda_{3,4}^{3}-\lambda_{2,4}^{2} \lambda_{1,2}^{3} ; \\
0 & =\lambda_{1,3}^{2} \lambda_{3,4}^{5}-\lambda_{2,4}^{2} \lambda_{1,2}^{5} ; & 0 & =\lambda_{1,2}^{3} \lambda_{2,3}^{5}-\lambda_{2,3}^{3} \lambda_{1,2}^{5} ; \\
0 & =\lambda_{1,2}^{3} \lambda_{2,4}^{5}+\lambda_{1,3}^{3} \lambda_{3,4}^{5}-\lambda_{2,4}^{3} \lambda_{1,2}^{5}-\lambda_{3,4}^{3} \lambda_{1,3}^{5} ; & 0 & =\lambda_{2,3}^{3} \lambda_{3,4}^{5}-\lambda_{3,4}^{3} \lambda_{2,3}^{5} .
\end{aligned}
$$

(5) We obtain the following subalgebra of $\mathfrak{g}_{4}$ isomorphic to $\mathfrak{n}_{5}^{4}$ :

$$
e_{1}=e_{3,4}, \quad e_{2}=e_{2,4}, \quad e_{3}=-\left(e_{2,3}+e_{1,2}\right), \quad e_{4}=e_{1,4}, \quad e_{5}=-e_{1,3} .
$$

Let us note that this representative of the abelian subalgebra has been chosen in a natural way, because it has only one basic vector with more than one summand.

\subsection{Nilpotent Lie Algebras of Dimensions less than 5}

By applying the method previously explained, the minimal dimension $\widehat{\mu}$ is obtained for each nilpotent Lie algebras of dimension less than 5. Besides, a representation with this dimension is given for each of these algebras in the following table which also shows the dimension $\mu$ (or an upper bound of it) given by Burde [3]:

\begin{tabular}{cccc}
\hline $\begin{array}{c}\text { Nilpotent } \\
\text { Lie algebra }\end{array}$ & $\begin{array}{c}\text { Dimension } \\
\mu\end{array}$ & $\begin{array}{c}\text { Dimension } \\
\widehat{\mu}\end{array}$ & $\begin{array}{c}\text { Minimal } \\
\text { representation }\end{array}$ \\
\hline $\mathfrak{n}_{1}^{1}$ & 0 & 2 & $\left\langle e_{1,2}\right\rangle$ \\
$\mathfrak{n}_{2}^{1}$ & 2 & 3 & $\left\langle e_{1,2}, e_{1,3}\right\rangle$ \\
$\mathfrak{n}_{3}^{1}$ & 3 & 3 & $\left\langle e_{1,2}, e_{1,3}, e_{2,3}\right\rangle$ \\
$\mathfrak{n}_{3}^{2}$ & 3 & 4 & $\left\langle e_{1,2}, e_{1,3}, e_{1,4}\right\rangle$ \\
$\mathfrak{n}_{4}^{1}$ & 4 & 4 & $\left\langle e_{1,2}, e_{1,3}, e_{1,4}, e_{2,3}+e_{3,4}\right\rangle$ \\
$\mathfrak{n}_{4}^{2}$ & 4 & 4 & $\left\langle e_{1,3}, e_{1,4}, e_{2,3}, e_{2,4}\right\rangle$ \\
$\mathfrak{n}_{4}^{3}$ & $\leq 5$ & 4 & $\left\langle e_{1,2}, e_{1,3}, e_{1,4}, e_{2,3}\right\rangle$ \\
\hline
\end{tabular}

The representations given in [5] for the algebras of dimension 2 and 3 belong to the type of those which we are studying. Besides, the dimension of these 
representations is equal to the minimal value obtained in this paper; however, in the same paper, the minimality of the representations was not studied in any sense. For dimension 4 , except for the filiform Lie algebra $\mathfrak{n}_{4}^{1}$, the representations given do not use the Lie algebras $\mathfrak{g}_{n}$ considered here. Besides, the minimality of such representations are not studied again.

\subsection{Nilpotent Lie Algebras of Dimension 5}

We conclude this paper by summing up the results obtained by applying the method previously explained to the 5-dimensional nilpotent Lie algebras. In the following table, we show both values $\mu$ and $\widehat{\mu}$ for all these Lie algebras. Note that the upper bounds of $\mu$ given by Burde [3] have been improved for most of these algebras:

\begin{tabular}{cccc}
\hline $\begin{array}{c}\text { Nilpotent } \\
\text { Lie algebra }\end{array}$ & $\begin{array}{c}\text { Dim. } \\
\mu\end{array}$ & $\begin{array}{c}\text { Dim. } \\
\mu\end{array}$ & $\begin{array}{c}\text { Minimal } \\
\text { representation }\end{array}$ \\
\hline $\mathfrak{n}_{5}^{1}$ & 5 & 5 & $\left\langle e_{1,2}, e_{1,3}, e_{1,4}, e_{1,5}, e_{2,3}+e_{3,4}+e_{4,5}\right\rangle$ \\
$\mathfrak{n}_{5}^{2}$ & 5 & 5 & $\left\langle e_{1,2}+e_{2,4}+e_{3,5}, e_{1,3}, e_{1,4}, e_{1,5}, e_{2,3}+e_{3,4}+e_{4,5}\right\rangle$ \\
$\mathfrak{n}_{5}^{3}$ & $\leq 6$ & 5 & $\left\langle e_{1,2}-e_{3,5}, e_{1,3}+e_{2,5}, e_{1,4}, e_{1,5}, e_{2,3}+e_{3,4}\right\rangle$ \\
$\mathfrak{n}_{5}^{7}$ & 4 & 5 & $\left\langle e_{1,3}, e_{1,4}, e_{1,5}, e_{2,4}, e_{2,5}\right\rangle$ \\
$\mathfrak{n}_{5}^{9}$ & $\leq 6$ & 5 & $\left\langle e_{1,2}, e_{1,3}, e_{1,4}, e_{1,5}, e_{2,3}\right\rangle$ \\
$\mathfrak{n}_{5}^{4}$ & $\leq 6$ & 4 & $\left\langle e_{1,2}+e_{2,3}, e_{1,3}, e_{1,4}, e_{2,4}, e_{3,4}\right\rangle$ \\
$\mathfrak{n}_{5}^{5}$ & $\leq 6$ & 4 & $\left\langle e_{1,2}, e_{1,3}, e_{1,4}, e_{2,3}, e_{2,4}\right\rangle$ \\
$\mathfrak{n}_{5}^{6}$ & 4 & 4 & $\left\langle e_{1,2}, e_{1,3}, e_{1,4}, e_{2,4}, e_{3,4}\right\rangle$ \\
$\mathfrak{n}_{5}^{8}$ & $\leq 6$ & 4 & $\left\langle e_{1,2}+e_{3,4}, e_{1,3}, e_{1,4}, e_{2,3}, e_{2,4}\right\rangle$ \\
\hline
\end{tabular}

With respect to the representations given in [5] for the 5-dimensional nilpotent Lie algebras, we can affirm that the minimality of these representations was not studied either, although most of these algebras are represented by the type of matrices considered in this paper. Moreover, some of these representations are not minimal. For example, a 5-dimensional representation is given for the nilpotent Lie algebras $\mathfrak{n}_{5}^{4}$ and $\mathfrak{n}_{5}^{5}$ by considering a Lie subalgebra of $\mathfrak{g}_{5}$ for each algebra. However, the minimal dimension to represent these two algebras is 4 by using two Lie algebras of $\mathfrak{g}_{4}$.

\section{REFERENCES}

1. Benjumea, J. C., Grupos de Lie asociados a álgebras de Lie filiformes, Ph.D. Thesis, Universidad de Sevilla, 2002.

2. Burde, D., Affine structures on nilmanifolds, Internat. J. Math. 7:5 (1996), 599-616. 
3. Burde, D., On a refinement of Ado's Theorem, Arch. Math. (Basel) 70 (1998), 118-127.

4. Fulton, W., and Harris, J., Representation Theory: a First Course, Springer-Verlag, New York, 1991.

5. Ghanam, R., Strugar, I., and Thompson, G., Matrix representations for low dimensional Lie algebras, Extracta Math. 20 (2005), 151-184.

6. Goze, M., and Khakimdjanov, Y., Nilpotent Lie Algebras, Kluwer Academic Publisher, Dordretch, The Netherlands, 1996.

7. Tenorio, A. F., Grupos de Lie asociados a álgebras de Lie nilpotentes, Ph.D. Thesis, Universidad de Sevilla, 2003.

8. Varadarajan, V. S., Lie Groups, Lie Algebras and Their Representations, Springer, New York, 1984.

DEPARTAMENTO DE GEOMETRÍA Y TOPOLOGÍA FACULTAD DE MATEMÁTICAS,

UNIVERSIDAD DE SEVILLA

APTDO. 1160

41080-SEVILLA

SPAIN

E-mail: jcbenjumea@us.es,jnvaldes@us.es
DPTO. ECONOMÍA, MÉTODOS CUANTITATIVOS E HISTORIA ECONÓMICA.

ESCUELA POLITÉCNICA SUPERIOR. UNIVERSIDAD PABLO DE OLAVIDE CTRA. UTRERA KM. 1 41013-SEVILLA SPAIN

E-mail: aftenorio@upo.es 